\title{
On higher-order flavour-singlet splitting and coefficient functions at large $x$
}

\author{
A. Vogt ${ }^{\mathrm{a}}$, G. Soar ${ }^{\mathrm{a}}$, S. Moch ${ }^{\mathrm{b}}$ and J.A.M. Vermaseren ${ }^{\mathrm{c}}$
}

aDepartment of Mathematical Sciences, University of Liverpool, Liverpool L69 3BX, United Kingdom

${ }^{\mathrm{b}}$ Deutsches Elektronensynchrotron DESY, Platanenallee 6, D-15738 Zeuthen, Germany

cNIKHEF, Science Park 105, 1098 XG Amsterdam, The Netherlands

We discuss the large- $x$ behaviour of the splitting functions $P_{\mathrm{qg}}$ and $P_{\mathrm{gq}}$ and of flavour-singlet coefficient functions, such as the gluon contributions $C_{2, \mathrm{~g}}$ and $C_{L, \mathrm{~g}}$ to the structure functions $F_{2, L}$, in massless perturbative QCD. These quantities are suppressed by one or two powers of $(1-x)$ with respect to the $(1-x)^{-1}$ terms which are the subject of the well-known threshold exponentiation. We show that the double-logarithmic contributions to $P_{\mathrm{qg}}$, $P_{\mathrm{gq}}$ and $C_{L}$ at order $\alpha_{\mathrm{s}}^{4}$ can be predicted from known third-order results and present, as a first step towards a full all-order generalization, the leading-logarithmic large- $x$ behaviour of $P_{\mathrm{qg}}, P_{\mathrm{gq}}$ and $C_{2, \mathrm{~g}}$ at all orders in $\alpha_{\mathrm{s}}$.

\section{Introduction}

Inclusive deep-inelastic lepton-nucleon scattering (DIS) via the exchange of a colour-neutral (gauge) boson, for the basic kinematics see Fig. 1, is a benchmark process of perturbative QCD.

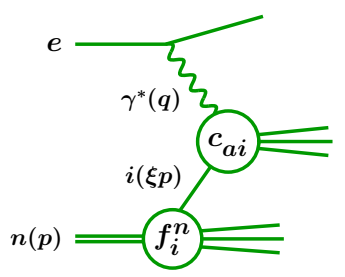

Scale, Bjorken variable

$$
\begin{aligned}
Q^{2} & =-q^{2} \\
x & =Q^{2} /(2 p \cdot q)
\end{aligned}
$$

Lowest order : $x=\xi$

Figure 1. The kinematics and perturbative QCD factorization of photon-exchange DIS.

Disregarding contributions suppressed by powers of $1 / Q^{2}$, the structure functions in electromagnetic DIS are given by

$$
x^{-1} F_{a}^{n}\left(x, Q^{2}\right)=\left[C_{a, i}\left(\alpha_{\mathrm{s}}\left(Q^{2}\right)\right) \otimes f_{i}^{n}\left(Q^{2}\right)\right](x)
$$

in terms of the coefficient functions $C_{a, i}, a=2, L$, $i=\mathrm{q}, \mathrm{g}$, and the nucleon parton distributions $f_{i}{ }^{n}$. Here $\otimes$ denotes the standard Mellin convolution, and the summation over $i$ is understood. Without loss of information, we identify the renormalization and factorization scale with the physical scale $Q^{2}$ in Eq. (1) and throughout this article.
The scale dependence of the parton densities is

$$
\frac{d f_{i}\left(\xi, \mu^{2}\right)}{d \ln Q^{2}}=\left[P_{i k}\left(\alpha_{\mathrm{s}}\left(Q^{2}\right)\right) \otimes f_{k}\left(Q^{2}\right)\right](\xi) .
$$

The coefficient functions in Eq. (1) and the splitting functions $P_{i k}$ can be expanded in powers of the strong coupling constant $a_{\mathrm{s}} \equiv \alpha_{\mathrm{s}} /(4 \pi)$,

$$
\begin{aligned}
C_{a, i}\left(x, \alpha_{\mathrm{s}}\right) & =\sum_{l=0} a_{\mathrm{s}}^{l+l_{a}} c_{a, i}^{(l)}(x), \\
P_{i k}\left(x, \alpha_{\mathrm{s}}\right) & =\sum_{l=0} a_{\mathrm{s}}^{l+1} P_{i k}^{(l)}(x)
\end{aligned}
$$

with $l_{a}=0$ for $F_{2}$ (and the Higgs-exchange structure function $F_{\phi}$ discussed below), and $l_{a}=1$ for the longitudinal structure function $F_{L}$. In this notation, the $\mathrm{N}^{n} \mathrm{LO}$ approximation includes the contributions with $l \leq n$ in both Eqs. (3) and (4).

The above (spin-averaged) splitting functions are presently known to $n=2[1,2]$, i.e., the nextto-next-to-leading order $\left(\mathrm{NNLO} \equiv \mathrm{N}^{2} \mathrm{LO}\right)$. The coefficient functions for the most important structure functions (including $F_{3}$ for charge-averaged $W$-exchange) have also been fully computed to order $\alpha_{\mathrm{s}}^{3}[3-5]$, while the less important chargeasymmetry $W$-cases are available only through a couple of low-integer Mellin- $N$ moments $[6,7]$. The frontier in the present massless case are now the $\alpha_{\mathrm{s}}^{4}$ corrections, for which first results have been obtained at the lowest value of $N[8,9]$. See Ref. [10] for the status of the third-order computation of the heavy-quark contributions to DIS. 


\section{The general large- $x$ behaviour}

We are interested in the leading contributions, in terms of powers in $(1-x)$, to Eqs. (3) and (4). The form of the diagonal splitting functions is stable under higher-order corrections in the $\overline{\mathrm{MS}}$ scheme, viz [11]

$$
P_{i i}^{(l)}=A_{i}^{(l)}(1-x)_{+}^{-1}+B_{i}^{(l)} \delta(1-x)+\ldots .
$$

The off-diagonal quantities, however, receive a double-logarithmic higher-order enhancement,

$$
P_{i \neq j}^{(l)}=\sum_{a=0}^{2 l} A_{i j, a}^{(l)} \ln ^{2 l-a}(1-x)+\ldots,
$$

where $A_{i j, a}^{(l)} \propto\left(C_{A}-C_{F}\right)^{l-a}$ for $a<l$ for (at least) $l \leq 2$ [2], i.e., all double logarithms vanish for $C_{F}=C_{A}$, which is part of the colour-factor choice leading to an $\mathcal{N}=1$ supersymmetric theory.

The leading large- $x$ parts of 'diagonal' coefficient functions, e.g., $C_{2, \mathrm{q}}$ and $C_{\phi, \mathrm{g}}$, are given by

$$
c_{\text {diag }}^{(l)}=\sum_{a=0}^{2 l-1} D_{i, a}^{(l)}\left[\frac{\ln ^{2 l-1-a}(1-x)}{1-x}\right]_{+}^{-1}+\ldots .
$$

These terms are resummed by the soft-gluon exponentiation [12-15]. For DIS structure functions (and some other semi-leptonic processes) this resummation is known at the next-to-next-to-nextto-leading logarithmic accuracy, i.e., the highest six logs are completely known to all orders [16].

No resummation has been derived so far for the off-diagonal (flavour-singlet) coefficient functions such as $C_{2, \mathrm{~g}}$ and $C_{\phi, \mathrm{q}}$ which are of the form

$$
c_{\text {off-d }}^{(l)}=\sum_{a=0}^{2 l-1} O_{i, a}^{(l)} \ln ^{2 l-1-a}(1-x)+\ldots .
$$

The coefficient functions for $F_{L}$ are suppressed by one power in $(1-x)$ with respect to those of $F_{2}$,

$$
c_{L, i}^{(l)}=\sum_{a=0}^{2 l} L_{L, i}^{(l)}(1-x)^{\delta_{i g}} \ln ^{2 l-a}(1-x)+\ldots
$$

recall our notation with $l_{L}=1$ in Eq. (3). The double-log contributions to $C_{L, \mathrm{q}}$ (and the $C_{F}=0$ part of $C_{L, \mathrm{~g}}$ ) have been resummed in Ref. [17], i.e., the respective highest three logarithms $(a=$ 0, 1 and 2 in Eq. (9)) are known to all orders.

Our aim is to derive corresponding predictions for all quantities in Eqs. (6), (8) and (9). The present contribution is a brief status report of this programme, which has not been finished so far.

\section{Physical evolution kernel for $\left(F_{2}, F_{\phi}\right)$}

The results of Ref. [17] and their extension to the non-leading corrections for $C_{2, \mathrm{q}}$ and other quantities at all orders in $(1-x)$ [18], see Ref. [19] for a brief summary, have been obtained by studying the non-singlet physical evolution kernels for the respective observables. It is thus natural to study also flavour-singlet physical kernels.

The most natural complement to the standard quantity $F_{2}$ with $c_{2, i}^{(0)}=\delta_{i q} \delta(1-x)$ is a structure function for a probe which directly interacts only with gluons, such as a scalar $\phi$ with a $\phi G^{\mu \nu} G_{\mu \nu}$ coupling to the gluon field [20]. In the Standard Model this interaction is realized for the Higgs boson in the limit of a heavy top-quark $[21,22]$. The coefficient functions $C_{\phi, i}$ have been determined recently in Refs. [23] and [24] to the second and third order in $\alpha_{\mathrm{s}}$, respectively.

We thus consider the 2-vector singlet structure function and $2 \times 2$ coefficient-function matrix

$$
F=\left(\begin{array}{c}
F_{2} \\
F_{\phi}
\end{array}\right), \quad C=\left(\begin{array}{cc}
C_{2, \mathrm{q}} & C_{2, \mathrm{~g}} \\
C_{\phi, \mathrm{q}} & C_{\phi, \mathrm{g}}
\end{array}\right) .
$$

With $P$ denoting the matrix of the splitting functions (7) and (8), the evolution kernel for $F$ reads

$$
\begin{aligned}
\frac{d F}{d \ln Q^{2}} & =\frac{d C}{d \ln Q^{2}} f+C P f \\
& =\left(\beta\left(a_{\mathrm{s}}\right) \frac{d C}{d a_{\mathrm{s}}} C^{-1}+C P C^{-1}\right) F \\
& =K F \quad \text { with } \quad K=\left(\begin{array}{ll}
K_{22} & K_{2 \phi} \\
K_{\phi 2} & K_{\phi \phi}
\end{array}\right) .
\end{aligned}
$$

$\beta\left(a_{\mathbf{s}}\right)=-\beta_{0} a_{\mathrm{s}}^{2}+\ldots$ with $\beta_{0}=11 C_{A} / 3-2 n_{f} / 3$ is the standard beta function of QCD. All products of $x$-dependent quantities have to be read as convolutions (or products of their Mellin transforms).

After expanding in $\alpha_{\mathrm{s}}$, the first term in the second line of Eq. (11) receives double-logarithmic contributions from the non-singlet and singlet coefficient functions (7) and (8). The second term, absent in the non-singlet cases of Refs. $[17,18]$, includes also the double-log terms of Eq. (6).

The crucial observation, proven by available three-loop calculations to order $\alpha_{\mathrm{s}}^{4}$ for the nonsinglet parts (thanks to Eq. (5)) and to order $\alpha_{\mathrm{s}}^{3}$ 
for the singlet contribution, is that the physical kernel $K$ is only single-log enhanced [24], i.e.,

$$
K_{a b}^{(l)}=\sum_{\eta=0}^{l} A_{a b, \eta}^{(l)}(1-x)^{-\delta_{a b}} \ln ^{l-\eta}(1-x)+\ldots
$$

where the expansion coefficients $K_{a b}^{(l)}$ are defined as in Eq. (4) for the splitting functions above.

We conjecture that also the flavour singlet part remains single-log enhanced at the fourth order. This implies a cancellation between the doublelogarithmic contributions to the, so far unknown, off-diagonal $l=3$ splitting functions (6) and the known $[4,24]$ coefficient functions to order $\alpha_{\mathrm{s}}^{3}$ from which the former can be deduced. The results are

$$
\begin{aligned}
& P_{\mathrm{qg}}^{(3)} / n_{f}=\ln ^{6}(1-x) \cdot 0 \\
&+ \ln ^{5}(1-x)\left[\frac{22}{27} C_{A F}^{3}-\frac{14}{27} C_{A F}^{2} C_{F}+\frac{4}{27} C_{A F}^{2} n_{f}\right] \\
&+\ln ^{4}(1-x)\left[\left(\frac{293}{27}-\frac{80}{9} \zeta_{2}\right) C_{A F}^{3}-\frac{116}{81} C_{A F}^{2} n_{f}\right. \\
&+\left(\frac{4477}{16}-8 \zeta_{2}\right) C_{A F}^{2} C_{F}-\frac{13}{81} C_{A F} C_{F}^{2} \\
&\left.+\frac{17}{81} C_{A F} C_{F} n_{f}-\frac{4}{81} C_{A F} n_{f}^{2}\right] \\
&+\mathcal{O}\left(\ln ^{3}(1-x)\right), \\
& P_{\mathrm{gq}}^{(3)} / C_{F}=\ln ^{6}(1-x) \cdot 0 \\
&+\ln ^{5}(1-x)\left[\frac{70}{27} C_{A F}^{3}-\frac{14}{27} C_{A F}^{2} C_{F}-\frac{4}{27} C_{A F}^{2} n_{f}\right] \\
&+\ln ^{4}(1-x)\left[\left(\frac{3280}{81}+\frac{16}{9} \zeta_{2}\right) C_{A F}^{3}-\frac{256}{27} C_{A F}^{2} n_{f}\right. \\
&+\left(\frac{637}{18}-8 \zeta_{2}\right) C_{A F}^{2} C_{F}-\frac{49}{81} C_{A F} C_{F}^{2} \\
&\left.+\frac{17}{81} C_{A F} C_{F} n_{f}+\frac{32}{81} C_{A F} n_{f}^{2}\right] \\
&+\mathcal{O}\left(\ln ^{3}(1-x)\right)
\end{aligned}
$$

with $C_{A F} \equiv C_{A}-C_{F}$. The vanishing of the leading $\ln ^{6}(1-x)$ contributions is due to a cancellation of contributions. Below we will address the question whether this cancellation is accidental or a structural feature. Eqs. (13) and (14) show the colour-factor pattern already noted for $l \leq 2$ below Eq. (6). The feature is not an obvious consequence of our derivation and can thus be viewed as a non-trivial check of the above conjecture.

The extension of the above results to all powers of $(1-x)$ can be found in Ref. [24].

\section{Physical evolution kernel for $\left(F_{2}, F_{L}\right)$}

The system of standard DIS structure functions

$$
F=\left(\begin{array}{c}
F_{2} \\
\widehat{F}_{L}
\end{array}\right), \quad \widehat{F}_{L}=F_{L} /\left(a_{\mathrm{s}} c_{L, \mathrm{q}}^{(0)}\right),
$$

studied before in Refs. [25,26], can be analyzed in complete analogy to the previous section. Our normalization of $\vec{F}_{L}$ (of course Eq. (15) involves a simple division only in Mellin- $N$ space) leads to

$$
C=\left(\begin{array}{cc}
\delta(1-x) & 0 \\
\delta(1-x) & \hat{c}_{L, \mathrm{~g}}^{(0)}
\end{array}\right)+\sum_{l=1} a_{\mathrm{s}}^{l}\left(\begin{array}{cc}
c_{2, \mathrm{q}}^{(l)} & c_{2, \mathrm{~g}}^{(l)} \\
\hat{c}_{L, \mathrm{q}}^{(l)} & \hat{c}_{L, \mathrm{~g}}^{(l)}
\end{array}\right) .
$$

The resulting elements of the physical kernel

$$
K=\left(\begin{array}{ll}
K_{22} & K_{2 \mathrm{~L}} \\
K_{\mathrm{L} 2} & K_{\mathrm{LL}}
\end{array}\right)
$$

are again single-log enhanced at large $x$ and read

$$
K_{a b}^{(l)}=\sum_{\eta=0}^{l} \widehat{A}_{a b, \eta}^{(l)}(1-x)^{-1} \ln ^{l-\eta}(1-x)+\ldots
$$

at, at least, $l \leq 3$ for the upper row of Eq. (17), with $\widehat{A}_{2 \mathrm{~L}, 0}^{(l)}=0$, and at $l \leq 2$ for the lower row.

Conjecturing that this behaviour holds at $l=3$ also for $K_{L 2}$ and $K_{L L}$, the three-loop results of Refs. [1-4] together with Eq. (13) yield

$$
\begin{aligned}
& c_{L, \mathrm{q}}^{(3)} / C_{F}= \ln ^{6}(1-x) \frac{16}{3} C_{F}^{3} \\
&+ \ln ^{5}(1-x)\left[\left(72-64 \zeta_{2}\right) C_{F}^{3}+\frac{80}{9} C_{F}^{2} n_{f}\right. \\
&\left.\quad-\left(\frac{728}{9}-32 \zeta_{2}\right) C_{F}^{2} C_{A}\right] \\
&+ \ln ^{4}(1-x) \cdot[\text { known coefficients }] \\
&+ \mathcal{O}\left(\ln ^{3}(1-x)\right), \\
& c_{L, \mathrm{~g}}^{(3)} / n_{f}=(1-x) \ln ^{6}(1-x) \frac{32}{3} C_{A}^{3} \\
&+(1-x) \ln ^{5}(1-x)\left[-\frac{2080}{9} C_{A}^{3}+\frac{64}{9} C_{A}^{2} n_{f}\right. \\
&\left.+\frac{104}{3} C_{A}^{2} C_{F}+\frac{40}{3} C_{F}^{3}\right] \\
&+(1-x) \ln ^{4}(1-x)\left[\left(\frac{70760}{27}-352 \zeta_{2}\right) C_{A}^{3}\right. \\
&+\left(\frac{25306}{27}-\frac{320}{3} \zeta_{2}\right) C_{A}^{2} C_{F}-\frac{4192}{27} C_{A}^{2} n_{f}
\end{aligned}
$$




$$
\begin{aligned}
& +\left(\frac{1600}{27}+32 \zeta_{2}\right) C_{A} C_{F}^{2}+\frac{556}{27} C_{A} C_{F} n_{f} \\
& \left.+\frac{32}{27} C_{A} n_{f}^{2}+\left(38-\frac{320}{3} \zeta_{2}\right) C_{F}^{3}+\frac{308}{27} C_{F}^{2} n_{f}\right] \\
& +\mathcal{O}\left((1-x) \ln ^{3}(1-x)\right),
\end{aligned}
$$

where the coefficient of $\ln ^{4}(1-x)$ in Eq. (19) has been suppressed for brevity. The complete form of this equation has been given in Ref. [17], where it was derived in another manner which did not involve the off-diagonal splitting functions. Consequently the consistency of the two derivations provides another confirmation of the correctness of the above result for $P_{\mathrm{qg}}^{(3)}$. The non- $C_{F}$ parts of Eq. (20) - here, as App. C of Ref. [24], given for $W$-exchange, i.e., without the $f l_{11}^{g}$ contribution for the photon case [4] - have also been derived, but not explicitly written down, in Ref. [17].

\section{Unfactorized off-diagonal amplitudes}

The single-log enhancement of the above physical kernels suggests an iterative structure of the unfactorized structure functions (forward amplitudes), from which the splitting and coefficient functions are obtained in Mellin- $N$ space via the mass-factorization relations

$$
T_{a, j}=\widetilde{C}_{a, i} Z_{i j}, \quad-\gamma \equiv P=\frac{d Z}{d \ln Q^{2}} Z^{-1}
$$

The $D$-dimensional coefficient functions $\widetilde{C}_{a}$ include terms with $\varepsilon^{k}, k \geq 0$ in dimensional regularization with $D=4-2 \varepsilon$. The transition functions $Z$ collect all terms which are singular for $\varepsilon \rightarrow 0$. Inverting the second relation in Eq. (21) yields

$$
\begin{aligned}
& \left.Z\right|_{a_{\mathrm{s}}^{n}}=\frac{1}{\varepsilon^{n}} \frac{\gamma_{0}^{n}}{n !}+\ldots \\
& \quad+\frac{1}{\varepsilon^{2}}\left(\frac{\gamma_{0} \gamma_{n-2}}{n(n-1)}+\frac{\gamma_{n-2} \gamma_{0}}{n}+\ldots\right)+\frac{1}{\varepsilon} \frac{\gamma_{n-1}}{n} .
\end{aligned}
$$

At order $\alpha_{\mathrm{s}}^{n}$, the $\varepsilon^{-n} \ldots \varepsilon^{-2}$ contribution to $T_{a}$ are given in terms of lower-order terms. The $\varepsilon^{-n}$ and $\varepsilon^{0}$ coefficients include the $n$-loop splitting functions and (four-dimensional) coefficient functions $C_{a}$, respectively. Terms with $\varepsilon^{k}, 0<k<l$ are required for the factorization at order $n+l$.

We now focus on the leading-logarithmic (LL) contributions to the off-diagonal quantities $T_{\phi, \mathrm{q}}$ and $T_{2, \mathrm{~g}}$ and summarize the results of Ref. [27].
With $L \equiv \ln N$ these terms are of the form

$$
\begin{aligned}
T_{\phi, \mathrm{q}}^{(n)} / C_{F} & \stackrel{\mathrm{LL}}{=} T_{2, \mathrm{~g}}^{(n)} / n_{f} \stackrel{\mathrm{LL}}{=} \frac{L^{n-1}}{N \varepsilon^{n}} \sum_{k=0}^{\infty}(\varepsilon L)^{k} \mathcal{L}_{n, k} . \\
\cdot & \left(C_{F}^{n-1}+C_{F}^{n-2} C_{A}+\ldots+C_{A}^{n-1}\right),(23)
\end{aligned}
$$

i.e., the coefficients $\mathcal{L}_{n, k}$ are the same for both amplitudes and all combinations of $C_{F}$ and $C_{A}$. Consequently an all-order relation for one colour structure of either amplitude is sufficient. The calculations of Ref. $[2,24]$ imply such a relation,

$$
\left.T_{\phi, \mathrm{q}}\right|_{C_{F} \text { only }} \stackrel{\mathrm{LL}}{=} T_{\phi, \mathrm{q}}^{(1)} \frac{\exp \left(a_{\mathrm{s}} T_{2, \mathrm{q}}^{(1)}\right)-1}{T_{2, \mathrm{q}}^{(1)}},
$$

in terms of the first-order expressions known to all powers of $\varepsilon$,

$$
\begin{aligned}
T_{\phi, \mathrm{q}}^{(1)} & \stackrel{\mathrm{LL}}{=}-\frac{2 C_{F}}{N} \frac{1}{\varepsilon} \exp (\varepsilon \ln N), \\
T_{2, \mathrm{q}}^{(1)} & \stackrel{\mathrm{LL}}{=} 4 C_{F} \frac{1}{\varepsilon^{2}}(\exp (\varepsilon \ln N)-1) .
\end{aligned}
$$

After carrying out the mass factorization to a very high order in $\alpha_{\mathrm{s}}$ (using Form [28]), the all-order analytic expressions for the leadinglogarithmic contributions to the splitting functions and coefficient functions have been derived. The former quantities are given by

$$
P_{\mathrm{qg}}^{\mathrm{LL}}\left(N, \alpha_{\mathrm{s}}\right)=\frac{n_{f}}{N} \frac{\alpha_{\mathrm{s}}}{2 \pi} \mathcal{B}_{0}\left(\tilde{a}_{\mathrm{s}}\right)
$$

with

$$
\begin{aligned}
\mathcal{B}_{0}(x) & =\sum_{n=0}^{\infty} \frac{B_{n}}{(n !)^{2}} x^{n} \\
& =1-\frac{x}{2}-\sum_{n=1}^{\infty} \frac{(-1)^{n}}{(2 n) !^{2}}\left|B_{2 n}\right| x^{2 n}, \\
\tilde{a}_{\mathrm{s}}= & \alpha_{\mathrm{s}} / \pi\left(C_{A}-C_{F}\right) \ln ^{2} N .
\end{aligned}
$$

The coefficients $B_{n}$ in Eq. (28) are the Bernoulli numbers in the standard normalization of Ref. [29]. The corresponding result for $P_{\mathrm{gq}}$ is obtained by replacing $n_{f}$ by $C_{F}$ in Eq. (27), and exchanging $C_{A}$ and $C_{F}$ in Eq. (29).

Due to $B_{2 n+1}=0$ for $n \geq 1$, the LL coefficients vanish at all even orders in $\alpha_{\mathrm{s}}$. Consequently the first lines of Eqs. (13) and (14) (recall the power counting (4)) are not at all accidental. 
The corresponding all-order result for the coefficient function $C_{2, \mathrm{~g}}$ reads

$$
C_{2, \mathrm{~g}}^{\mathrm{LL}}\left(N, \alpha_{\mathrm{s}}\right)=\frac{1}{2 N \ln N} \frac{n_{f}}{C_{A}-C_{F}} .
$$

$\cdot\left\{\exp \left(2 C_{F} a_{\mathbf{s}} \ln ^{2} N\right) \mathcal{B}_{0}\left(\tilde{a}_{\mathrm{s}}\right)-\exp \left(2 C_{A} a_{\mathbf{s}} \ln ^{2} N\right)\right\}$

$C_{\phi, q}^{\mathrm{LL}}$ can be obtained from this result by a simple substitution of colour factors.

Inserting Eqs. (5) (only the lowest-order terms with $A_{q}^{(0)}=4 C_{F}$ and $A_{g}^{(0)}=4 C_{A}$ contribute), (27), (30), and the well-known relation [30-32]

$$
C_{2, \mathrm{q}} \stackrel{\mathrm{LL}}{=} \exp \left(2 a_{\mathrm{s}} C_{F} \ln ^{2} N\right)
$$

and its analogue for $C_{\phi, \mathrm{g}}$ into the physical kernel $K_{2 \phi}$ in Eq. (11), one finds that the highest double logarithm indeed vanishes at all orders in $\alpha_{\mathrm{s}}$. The same is found for $K_{\phi 2}$. Hence the amplitudebased resummation verifies the conjecture made below Eq. (12) for $a \neq b$, if presently only for the leading double logarithm.

The function $\mathcal{B}_{0}(x)$ in Eq. (28) appears to be a new function. The relation between $\left|B_{2 n}\right|$ in the second line and the even values of Riemann's $\zeta$-function [29] implies that this series converges for all values of $x$. At positive $x$ the even part of $\mathcal{B}_{0}$ compensates the odd $-x / 2$ contribution up to an oscillation around zero, which persists, in an increasingly irregular manner, at very large (and possibly all) values of $x$ [33].

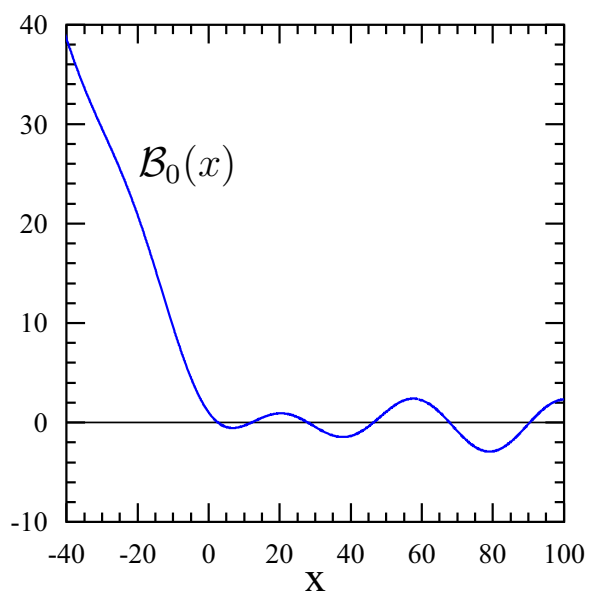

Figure 2. The function $\mathcal{B}_{0}(x)$ in Eq. (28), evaluated using its defining Taylor expansion.

\section{Summary and Outlook}

We have summarized the status of our large- $x$ predictions of higher-order off-diagonal splitting functions and DIS coefficient functions. The coefficients of the highest three powers of $\ln (1-x)$ have been derived for the four-loop contributions to the splitting functions $P_{\mathrm{qg}}$ and $P_{\mathrm{gq}}$ from the three-loop coefficient functions and the singlelogarithmic enhancement of the physical evolution kernel for the system $\left(F_{2}, F_{\phi}\right)$ of flavoursinglet structure functions at order $\alpha_{\mathrm{s}}^{4}[24]$. In the present contribution we have employed these results to derive also the leading three large- $x$ logarithms for the fourth-order gluon coefficient function $C_{L, \mathrm{~g}}$ for the longitudinal structure function from the analogous kernel for $\left(F_{2}, F_{L}\right)$.

These results will become phenomenologically relevant, via effective $x$-space parametrizations analogous to, e.g., those of Ref. [34], once the next major step towards a full fourth-order calculation of deep-inelastic scattering, the extension of Ref. [35] to order $\alpha_{\mathrm{s}}^{4}$, has been taken.

The determination of flavour-singlet quantities from the physical kernels is neither rigorous, nor - unlike in flavour non-singlet cases $[17,18]$ - can it be extended to all orders in $\alpha_{\mathrm{s}}$. We have presented first all-order leading-logarithmic results of a rigorous and more powerful approach, the prediction of the coefficients of the highest double logarithms from the $D$-dimensional structure of the unfactorized structure functions together with mass-factorization to all orders [27].

We expect that, similar to the non-singlet case, the all-order resummation of double-logarithmic large- $x$ contributions to flavour-singlet quantities can be extended beyond parton evolution and inclusive DIS. For example, the leading-logarithmic results of Ref. [27] can be carried over directly to semi-inclusive electron-positron annihilation and $Z$ - or Higgs-boson decay. On the other hand, we do not foresee an extension of our results to single-logarithmically enhanced large- $x$ terms. It will be interesting to see whether such an extension can be achieved in alternative approaches such as the application of soft-collinear effective theory to large- $x$ DIS [36-38] or the recent pathintegral formulation of Ref. [39]. 


\section{REFERENCES}

1. S. Moch, J.A.M. Vermaseren and A. Vogt, Nucl. Phys. B688 (2004) 101, hep-ph/0403192

2. A. Vogt, J.A.M. Vermaseren and S. Moch, Nucl. Phys. B691 (2004) 129, hep-ph/0404111

3. S. Moch, J.A.M. Vermaseren and A. Vogt, Phys. Lett. B606 (2005) 123, hep-ph/0411112

4. J.A.M. Vermaseren, A. Vogt and S. Moch, Nucl. Phys. B724 (2005) 3, hep-ph/0504242

5. S. Moch, J.A.M. Vermaseren, A. Vogt, Nucl. Phys. B813 (2009) 220, arXiv:0812.4168

6. S. Moch and M. Rogal, Nucl. Phys. B782 (2007) 51, arXiv:0704.1740

7. S. Moch, M. Rogal and A. Vogt, Nucl. Phys. B790 (2008) 317, arXiv:0708.3731

8. P.A. Baikov and K.G. Chetyrkin, Nucl. Phys. B (Proc. Suppl.) 160 (2006) 76

9. P. Baikov, K. Chetyrkin, J. Kühn, Phys. Rev. Lett. 104 (2010) 132004, arXiv:1001.3606

10. I. Bierenbaum, J. Blümlein and S. Klein, Nucl. Phys. B820 (2009) 417, arXiv:0904.3563

11. G.P. Korchemsky, Mod. Phys. Lett. A4 (1989) 1257

12. G. Sterman, Nucl. Phys. B281 (1987) 310

13. L. Magnea, Nucl. Phys. B349 (1991) 703

14. S. Catani and L. Trentadue, Nucl. Phys. B327 (1989) 323; ibid. B353 (1991) 183

15. S. Catani, M.L. Mangano, P. Nason and L. Trentadue, Nucl. Phys. B478 (1996) 273, hep$\mathrm{ph} / 9604351$

16. S. Moch, J.A.M. Vermaseren and A. Vogt, Nucl. Phys. B726 (2005) 317, hep-ph/0506288

17. S. Moch and A. Vogt, JHEP 0904 (2009) 081, arXiv:0902.2342

18. S. Moch and A. Vogt, JHEP 0911 (2009) 099, arXiv:0909.2124

19. A. Vogt, S. Moch, G. Soar and J. Vermaseren, PoS RADCOR 2009, 053, arXiv:1001.3554

20. W. Furmanski and R. Petronzio, Z. Phys. C11 (1982) 293

21. J.R. Ellis, M.K. Gaillard and D.V. Nanopoulos, Nucl. Phys. B106 (1976) 292

22. M. Shifman, A. Vainshtein, M. Voloshin and V. Zakharov, Sov. J. Nucl. Phys. 30 (1979) 711

23. A. Daleo, A. Gehrmann-De Ridder, T. Gehrmann and G. Luisoni, JHEP 1001 (2010) 118, arXiv:0912.0374
24. G. Soar, S. Moch, J. Vermaseren and A. Vogt, Nucl. Phys. B832 (2010) 152, arXiv:0912.0369

25. S. Catani, Z. Phys. C75 (1997) 665, hep-ph/ 9609263

26. J. Blümlein, V. Ravindran, W. van Neerven, Nucl. Phys. B586 (2000) 349, hep-ph/0004172

27. A. Vogt, Phys. Lett. B691 (2010) 77, arXiv: 1005.1606

28. J.A.M. Vermaseren, New features of FORM, math-ph/0010025;

29. M. Abramowitz and I.A. Stegun (eds.), Handbook of Mathematical Functions, Dover (New York) 1965

30. G. Parisi, Phys. Lett. B90 (1980) 295

31. G. Curci, M. Greco, Phys. Lett. B92 ('80) 175

32. D. Amati, A. Bassetto, M. Ciafaloni, G. Marchesini and G. Veneziano, Nucl. Phys. B173 (1980) 429

33. D. Broadhurst, private communication

34. W.L. van Neerven and A. Vogt, Phys. Lett. B490 (2000) 111, hep-ph/0007362

35. S.A. Larin, P. Nogueira, T. van Ritbergen and J. Vermaseren, Nucl. Phys. B492 (1997) 338, hep-ph/9605317

36. A.V. Manohar, Phys. Rev. D68 (2003) 114019, hep-ph/0309176

37. A. Idilbi, X.D. Ji and F. Yuan, Nucl. Phys. B753 (2006) 42, hep-ph/0605068

38. T. Becher, M. Neubert, B.D. Pecjak, JHEP 0701 (2007) 076, arXiv:hep-ph/0607228

39. E. Laenen, G. Stavenga and C.D. White, JHEP 0903 (2009) 054, arXiv:0811.2067 